\title{
Disorder and the quantum critical point in $\mathrm{UCu}_{5-x} \mathbf{P d}_{x}$
}

\author{
R Chau $\uparrow \S$, M C Aronson‡, E J Freeman $\dagger$ and M B Maple $\dagger$ \\ $\dagger$ Department of Physics and the Institute for Pure and Applied Physical Sciences, University of \\ California, San Diego, CA 92093, USA \\ \$ The Harrison M Randall Laboratory of Physics, University of Michigan, Ann Arbor, \\ MI 48109-1120, USA
}

Received 2 February 2000

\begin{abstract}
We have measured the magnetization of $\mathrm{UCu}_{5-x} \operatorname{Pd}_{x}(x=0.7,1,1.5,2)$ in fields $H$ as large as $30 \mathrm{~T}$ and for temperatures between $1.4 \mathrm{~K}$ and $150 \mathrm{~K}$. A composition- and temperaturedependent characteristic field $H^{*}(x, T)$ is identified, which separates two different non-Fermi liquid scaling regimes, present even in zero field. For $H \geqslant H^{*}(x, T)$, composition-independent scaling is found, while for $H \leqslant H^{*}(x, T)$, different exponents are found for each composition. This second phase is centred in temperature and composition around the $x=1, T=0$ critical point, and we argue that it is consistent with the presence of a Griffiths phase.
\end{abstract}

In recent years, it has been discovered that a zero-temperature critical point can be induced in a variety of metallic magnets by using pressure or compositional variation to suppress magnetic ordering temperatures to zero. The properties of this novel phase transition have prompted much experimental and theoretical work [1,2]. The fundamental excitations near such quantum critical points lead to unusual temperature and energy dependences in measured quantities, which are said to display non-Fermi liquid (nFL) behaviour. There is mounting evidence that disorder plays an important role in these systems, especially very near the $T=0$ critical point. However, there is little consensus about how profoundly disorder and nFL behaviour are interrelated, although a variety of models have been proposed. The Kondo disorder model argues that the nFL behaviour is associated with a distribution of Kondo temperatures $T_{K}$ due to disorder, with no need to invoke long-range magnetic correlations [3,4]. More recently, it has been proposed that nFL behaviour has its roots in the critical phenomena of the $T=0$ magnetic transition, with disorder limiting the growth of magnetic correlations, similar in spirit to the case of finite temperature phase transitions [5,6]. Finally, it has been proposed that nFL behaviour is an intrinsic feature of a $T=0$ magnet, and for much of the magnetic phase diagram is essentially unaffected by the presence of modest amounts of disorder $[7,8]$.

Experimentally, the resolution of this conflict must lie with delineating parts of the phase diagram over which the magnetic correlations develop freely and those where they are limited by disorder. This requires the formulation of an experimental criterion for such a crossover, which we present here. Given this criterion, it will be possible to revisit the existing scaling studies [8-10] to assess whether the nFL behaviour is limited to the disorder-dominated regime or whether it is an intrinsic feature associated with the $T=0$ critical point. We have studied the scaling properties of the magnetization of $\mathrm{UCu}_{5-x} \mathrm{Pd}_{x}$ over a very broad range of fields and temperatures for compositions both near and far from the critical composition. We confirm that

$\S$ Present address: Lawrence Livermore National Laboratory, Livermore, CA 94550, USA. 
two different scaling behaviours are present, and that disorder interacts with residual magnetic correlations in a subtle way very near the critical point. However, our experiments show unambiguously that for much of the temperature-composition phase diagram, the intrinsic critical phenomena of the $T=0$ magnetic transition can be observed.

We chose the $\mathrm{UCu}_{5-x} \mathrm{Pd}_{x}$ system to probe the nature of $\mathrm{nFL}$ scaling because its phase diagram and electronic properties have been extensively explored, and the degree of disorder has been carefully quantified for the compositions $x=1$ and $1.5[3,9,11,13,14]$. Inelastic neutron scattering measurements show that magnetic excitations with energies less than $25 \mathrm{meV}$ but greater than $\sim 2 \mathrm{meV}$ are $\mathrm{nFL}$-like; i.e., that the imaginary part of the dynamical susceptibility $\chi^{\prime \prime}(\omega, T)=T^{1 / 3} \mathcal{Z}(\omega / T)$ [15]. A Kramers-Kronig analysis [8] demonstrated that these excitations are responsible for the temperature dependence of the static magnetic susceptibility, $\chi(T) \propto T^{-1 / 3}$, found in measurements above $T^{*}=40 \mathrm{~K}$ for $\mathrm{UCu}_{4} \mathrm{Pd}$ and $T^{*}=10 \mathrm{~K}$ for $\mathrm{UCu}_{3.5} \mathrm{Pd}_{1.5}$. In the same temperature range in both compounds, the electrical resistivity $\rho(T) \propto T^{1 / 3}$, as expected for the scattering of quasiparticles from nFL excitations of individual uranium ions [16].

There is substantial experimental evidence for both $\mathrm{UCu}_{4} \mathrm{Pd}$ and $\mathrm{UCu}_{3.5} \mathrm{Pd}_{1.5}$ that a different type of nFL behaviour develops at the lowest temperatures. For $T<T^{*}$, a different set of temperature dependences are observed for the two compositions $[9,11]$. The magnetic susceptibility varies as $\chi(T) \propto T^{-0.28}$ for $\mathrm{UCu}_{4} \mathrm{Pd}$ and $\chi(T) \propto T^{-0.25}$ for $\mathrm{UCu}_{3.5} \mathrm{Pd}_{1.5}$, respectively, while $\rho(T)$ exhibits a linear temperature dependence for both compositions. Much less is known about this regime, as no direct information about the scaling properties of the excitations with energies less than $T^{*}$ is available yet from inelastic neutron scattering measurements. We have used the field and temperature scaling properties of the magnetization to determine $T^{*}(x)$ and demonstrate that the critical behaviours for $T \leqslant T^{*}$ and $T \geqslant T^{*}$ are distinct.

Spherical, polycrystalline samples of $\mathrm{UCu}_{5-x} \mathrm{Pd}_{x}(x=0.7,1,1.5,2)$ were prepared by a conventional arc-melting technique. Magnetization measurements were performed with a Lakeshore VSM using fields between $2000 \mathrm{G}$ and $30 \mathrm{~T}$ in a Bitter magnet at the National High Magnetic Field Laboratory, calibrated with a Ni standard. The data are corrected for demagnetization effects, and the very small contributions from the sample holder and secondary coils have been subtracted. Magnetization curves $M(H)$ for $\mathrm{UCu}_{4.3} \mathrm{Pd}_{0.7}$ taken at temperatures between $1.4 \mathrm{~K}$ and $70 \mathrm{~K}$ are shown in figure 1 and are similar to those found for the other compositions studied, $x=1,1.5,2$. For each composition, $M(H)$ is nonlinear at the lowest temperatures. The temperature dependence of the leading-order nonlinear susceptibility $\chi_{3}$ determined from magnetization sweeps for each compound is shown in the inset of figure 1. The observation that $\chi_{3}(T)$ is large and negative indicates a magnetic doublet ground state with a low characteristic temperature for moment screening. Further, the insensitivity of $\chi_{3}(T)$ to composition suggests that the crystal-field levels are only weakly modified with Pd doping.

Since the magnetization in a given field decreases with increasing temperature, we scaled the magnetization curves in the generic form expected for a non-Fermi liquid [7]: $(M / H) T^{\gamma}=\mathcal{F}\left(H / T^{\beta+\gamma}\right)$. Two different $\mathrm{nFL}$ scaling regimes are observed in $\mathrm{UCu}_{5-x} \mathrm{Pd}_{x}$ for $0.7 \leqslant x \leqslant 1.5$. Figure 2 shows the $H-T$ scaling for $\mathrm{UCu}_{4.3} \mathrm{Pd}_{0.7}$, which yields the exponents $\gamma=0.35 \pm 0.02$ and $\beta+\gamma=0.95 \pm 0.02$. At a given temperature, this scaling is found only above a characteristic field, $H^{*}(T)$, indicated in figure 2 by the arrows. The field where this scaling breaks down at each temperature defines $H^{*}(T)$. The temperature dependence of $H^{*}$ for $x=0.7,1,1.5$ is plotted in the inset of figure 2. At each composition, $H^{*}$ decreases approximately linearly with increasing temperature and vanishes at a zero-field crossover temperature $T^{*}(x)$, which is $5 \mathrm{~K}$ for $x=0.7,28 \mathrm{~K}$ for $x=1$, and $13 \mathrm{~K}$ for $x=1.5$. We will argue that the line $H^{*}(T)$ represents a crossover scale between two types of scaling. 


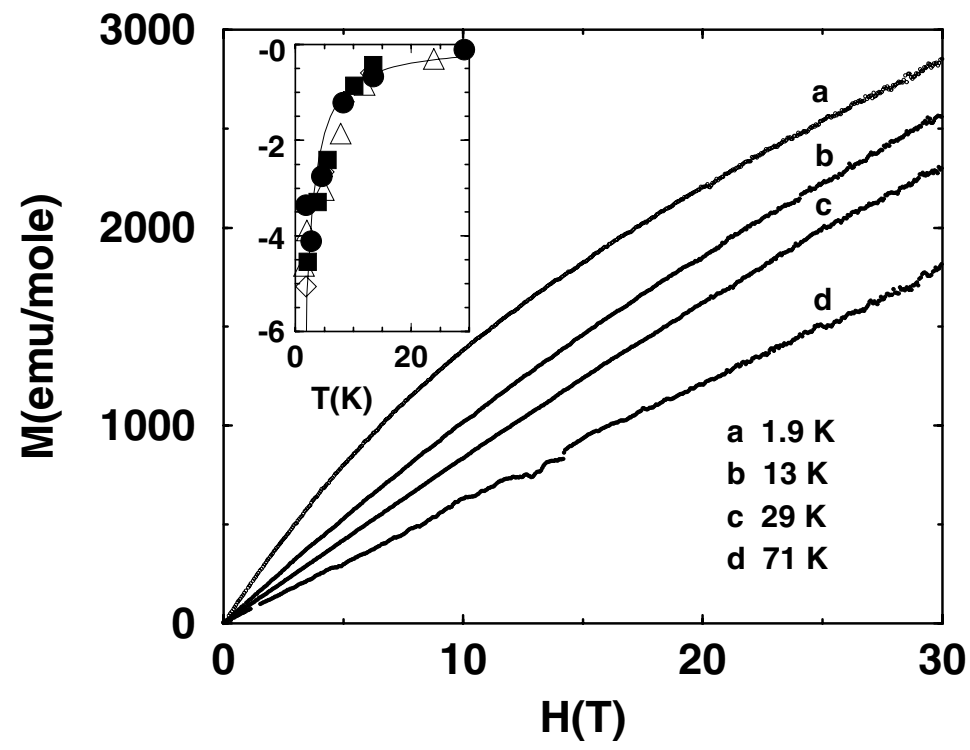

Figure 1. Magnetization $M$ versus applied magnetic field $H$ at various fixed temperatures for $\mathrm{UCu}_{4.3} \mathrm{Pd}_{0.7}$. Inset: the temperature dependence of the leading nonlinear susceptibility $\chi_{3}(T)$ for $\mathrm{UCu}_{5-x} \mathrm{Pd}_{x}$ with $x=0.7(\bullet), 1(\square), 1.5(\diamond)$, and $2(\triangle)$. The solid line is the best power-law fit, $\chi_{3} \propto T^{-\alpha}$, with $\alpha=1.1 \pm 0.1$.

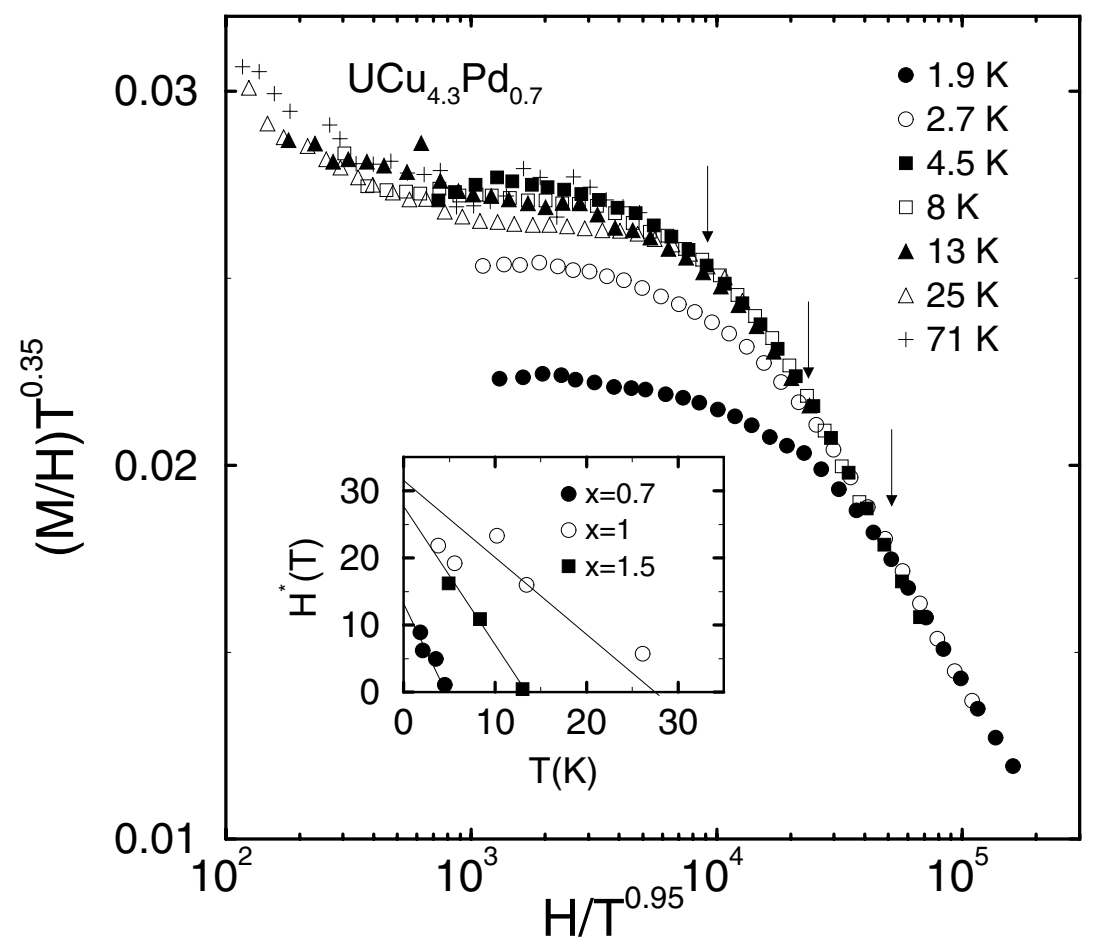

Figure 2. The scaling plot of $(M / H) T^{0.35}$ versus $H / T^{0.95}$ for $\mathrm{UCu}_{4.3} \mathrm{Pd}_{0.7}$. The data above a composition- and temperature-dependent field $H^{*}$, indicated at each temperature by arrows, define a scaling function. For clarity, only every tenth point is shown at each temperature. The solid lines are guides to the eye. Inset: $H^{*}$ versus $T$ for $\mathrm{UCu}_{5-x} \mathrm{Pd}_{x}$ samples with $x=0.7,1$, and 1.5 . 
The high-field/high-temperature regime is distinguished by the composition independence of the scaling exponents. The scaled magnetization curves shown in figure 3(a), with the $H \leqslant H^{*}(T, x)$ data omitted, demonstrate that the same scaling relation is found for $H \geqslant H^{*}$ for $\mathrm{UCu}_{5-x} \mathrm{Pd}_{x}$ for $x=0.7,1$, and 1.5 , but not for spin-glass-ordered $\mathrm{UCu}_{3} \mathrm{Pd}_{2}$, where no scaling is found over any appreciable range of temperatures or fields. A qualitatively different scaling is found for fields less than $H^{*}(T, x)$. The scaled magnetization curves for $x=1$ and 1.5, constructed by removing all data with $H \geqslant H^{*}(T, x)$, appear in figure 3(b). In contrast to the scaling found in the high-field region, the scaling exponents in the low-field regions are composition dependent. The low-field scaling exponents are given in table 1 . The properties of the scaling function $\mathcal{F}$ are consistent with previously measured zero-field quantities. Specifically, figure 3 shows that in the limit of zero field, $\mathcal{F}(0) \rightarrow$ constant, which yields the temperature dependence observed for the magnetic susceptibility for $T \geqslant T^{*}, \chi(T)=\lim _{H \rightarrow 0}(M / H) \propto T^{-0.35}$, while in the high-field/lowtemperature limit, $\mathcal{F}(x) \rightarrow x^{-1 / 3}[8]$.
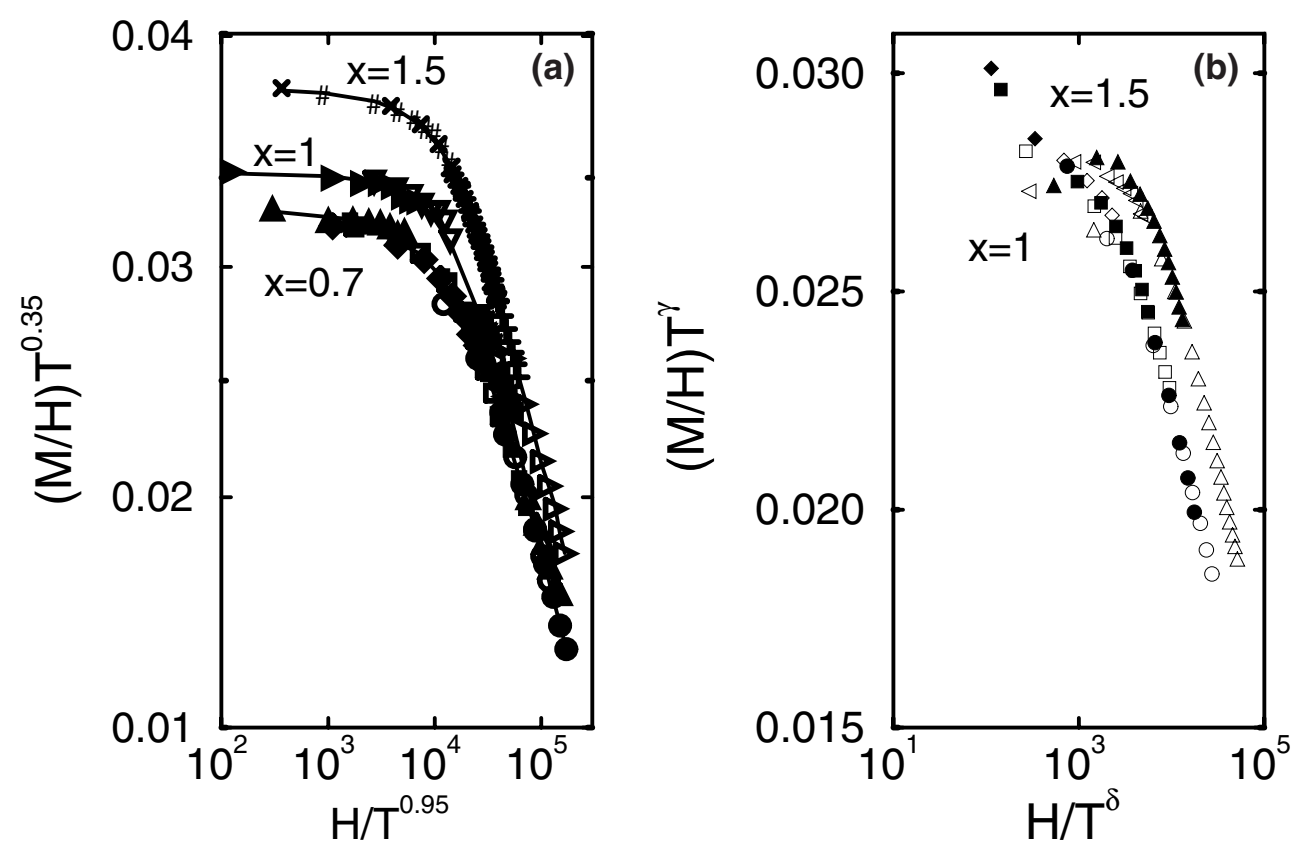

Figure 3. Demonstration of (a) composition-independent scaling of $M(H, T)$ for $H>H^{*}(T)$ and (b) composition-dependent scaling of $M(H, T)$ for $H<H^{*}(T)$. The symbols represent the following temperatures: $(\bullet) 1.8 \mathrm{~K} ;(\bigcirc) 2.1 \mathrm{~K} ;(\square) 4.5 \mathrm{~K} ;(\square) 8.2 \mathrm{~K} ;(\diamond) 13.4 \mathrm{~K} ;(\diamond) 29 \mathrm{~K}$; $(\Delta) 51 \mathrm{~K} ;(\triangle) 2.1 \mathrm{~K} ;(\triangleleft) 5.6 \mathrm{~K} ;(\triangleleft) 10.2 \mathrm{~K} ;(\boldsymbol{\nabla}) 13 \mathrm{~K} ;(\nabla) 26 \mathrm{~K} ;(\triangleright) 51 \mathrm{~K} ;(\triangleright) 1.8 \mathrm{~K} ;(+) 4.9 \mathrm{~K}$; (*) $8.4 \mathrm{~K}$; (×) $13 \mathrm{~K}$; (\#) $26 \mathrm{~K}$. The symbols have the same meaning in (a) and (b), although not every symbol appears in (b). For clarity, only every tenth point is shown at each temperature. The solid lines are guides to the eye.

Combining the magnetization scaling with previous measurements of $\rho(T), \chi(T)$, and specific heat $[9,11]$, we construct a new zero-field magnetic phase diagram of the $\mathrm{UCu}_{5-x} \mathrm{Pd}_{x}$ system which is presented in figure 4 . The antiferromagnetic phase line terminates at $x_{c} \sim 1$ where the low-field nFL scaling region extends over the broadest range of temperatures. This low-field $\mathrm{nFL}$ scaling regime spans the concentration range $(0.7 \leqslant x \leqslant 1.5)$, vanishes above the antiferromagnetic transition $T_{N}=3.35 \mathrm{~K}$ at $x=0.7$, and is totally absent at $x=2$. Lowtemperature $(T<1 \mathrm{~K})$ magnetic susceptibility measurements [12] reveal irreversibility in the 
Table 1. Scaling exponents are obtained from scaling the magnetization in both the high- and low-field regions for $\mathrm{UCu}_{5-x} \mathrm{Pd}_{x}(x=0.7,1,1.5)$. The high-field exponents are composition dependent while the low-field exponents are composition dependent.

\begin{tabular}{|c|c|c|c|c|}
\hline \multirow[b]{2}{*}{ Sample } & \multicolumn{2}{|c|}{ High field } & \multicolumn{2}{|c|}{ Low field } \\
\hline & $\gamma$ & $\gamma+\delta$ & $\gamma$ & $\gamma+\delta$ \\
\hline$x=0.7$ & $0.35 \pm 0.02$ & $0.95 \pm 0.02$ & 0.20 & 1.22 \\
\hline$x=1.0$ & $0.35 \pm 0.02$ & $0.95 \pm 0.02$ & 0.33 & 1.33 \\
\hline$x=1.5$ & $0.35 \pm 0.02$ & $0.95 \pm 0.02$ & 0.26 & 1.10 \\
\hline
\end{tabular}
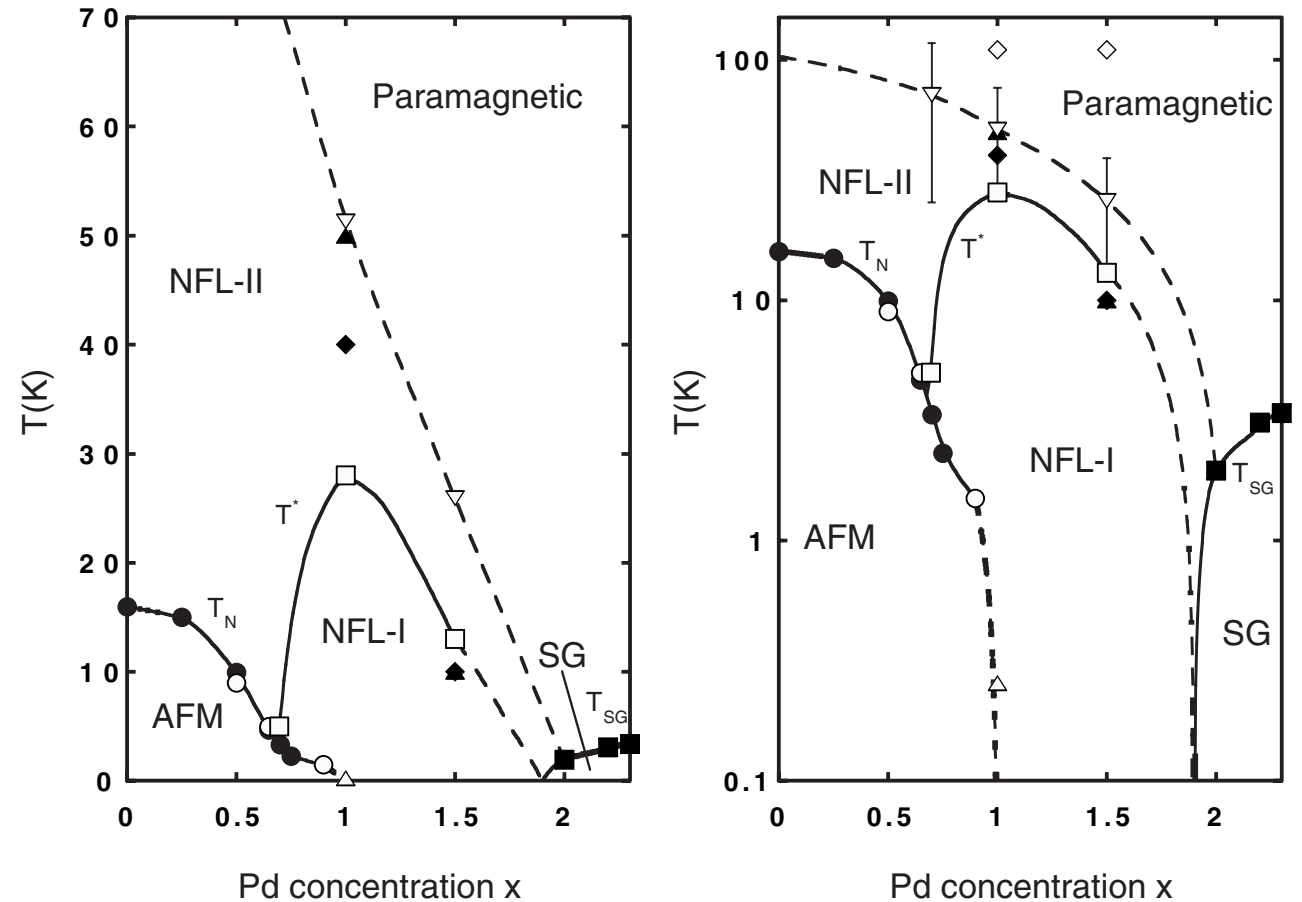

Figure 4. The phase diagram of $\mathrm{UCu}_{5-x} \mathrm{Pd}_{x}$ on (a) a linear $T$-scale and (b) a logarithmic $T$-scale. Néel temperatures taken from specific heat (open circles) and magnetic susceptibility (filled circles) measurements delineate the boundary of the antiferromagnetic phase for $x \leqslant 1$. Spin-glass freezing temperatures based on magnetic susceptibility measurements (filled squares) define the spin-glass phase. The open triangle is from the low- $T$ measurements of Vollmer et al [12]. The crossover line $T^{*}(x)$ based on the values of $T^{*}$ (open squares) inferred from magnetization scaling, magnetic susceptibility (filled diamonds), and electrical resistivity (filled triangle) is proposed to separate a Griffiths phase (NFL-I) from the homogeneous non-Fermi liquid phase (NFL-II). The open inverted triangles represent the lowest temperatures where paramagnetic behaviour was observed and loosely define the paramagnetic crossover line. The open diamonds indicate Kondo temperatures taken from inelastic neutron scattering measurements. The error bars represent uncertainty due to the limited number of temperatures sampled in determining the paramagnetic crossover. The solid lines are guides to the eye.

$\mathrm{dc}$ magnetization and frequency dependence in the ac susceptibility of $\mathrm{UCu}_{4} \mathrm{Pd}$ at $0.255 \mathrm{~K}$ which has been interpreted as spin-glass behaviour. This may indicate that the assumption of equilibrium dynamics may not be correct at the lowest temperatures. As the temperature is raised above $T \geqslant T^{*}(x)$, there is a crossover to a second type of nFL scaling in the concentration 
range $0.7 \leqslant x \leqslant 1.5$. The line $T^{*}(x)$, taken from the magnetization scaling, separates the two different $\mathrm{nFL}$ scaling regimes. Crossover temperatures inferred from zero-field $\rho(T)$ and $\chi(T)$ measurements $[9,11]$ are also indicated in figure 4 , and agree very well with $T^{*}(x)$. The second nFL scaling region is ultimately cut-off at higher temperature and crosses over to paramagnetic behaviour. The cut-off temperature indicated in figure 4 is defined from our magnetization measurements as the lowest temperature at which we are unable to resolve any nonlinearity in the $30 \mathrm{~T}$ magnetization sweeps. Measurements at higher fields are required to determine whether this is an independent temperature scale, or whether the characteristic field scale simply exceeds our experimental window. The Kondo temperature, taken from inelastic neutron scattering measurements, hardly changes with Pd concentration, and in agreement with the general scenario suggested by Doniach [17] remains finite in the vicinity of the quantum critical point.

For $x \geqslant 1.5$, we find that the paramagnetic behaviour extends to very low temperatures, eventually supplanting the second, higher-temperature $\mathrm{nFL}$ scaling region. Figure 4 shows that both nFL scaling regions are quickly suppressed at Pd concentrations $x>x_{c}=1$, suggesting that the quantum critical point associated with the vanishing of the spin-glass freezing temperature does not have a significant influence on the $\mathrm{nFL}$ behaviour. The magnetic phase diagram clearly associates the nFL behaviour in both regions I and II with the $x_{c}=1$, $T=0$ fixed point.

We propose that $\mathrm{nFL}$ scaling region I is a Griffiths phase, resulting from the interplay of residual disorder and finite-length-scale magnetic correlations. The long-time dynamics of the paramagnetic phase, sufficiently close to the $T=0$ critical point, is dominated by the slow reorientation of rare, magnetically ordered clusters, via quantum mechanical tunnelling $[5,6,19]$. An exponent $\lambda$ describes the power-law decay of the cluster magnetization, and controls the low-temperature dependences of the magnetic susceptibility and the specific heat: $\chi(T) \propto T^{-1+\lambda}$ and $C(T) \propto T^{\lambda}$. It is expected that $\lambda$ will be composition dependent, with its value reflecting the distributions in number, size, and magnetization of the clusters present in each sample. We believe that by including a range of possible cluster sizes and dynamics, the Griffiths phase scenario is more general and applicable to $\mathrm{UCu}_{5-x} \mathrm{Pd}_{x}$ than a previous description in which isolated magnetic impurities and a uniform response were used to model the nonlinearities in the magnetization [18].

There is currently no complete theory of the Griffiths phase in a magnetic field; however, in the limit $H \rightarrow 0, M / H=\chi(T)=T^{-1+\lambda}$. Table 2 shows $\lambda$-values for $x=0.7,1$, and 1.5, obtained from our magnetization scaling. These values are consistent with recently reported values from specific heat and dc magnetic susceptibility measurements [20]. The smallest value for $\lambda$ is found in both measurements for $x=x_{c}=1$, where the proposed Griffiths phase persists over the largest range of temperatures, $T \leqslant T^{*}=30 \mathrm{~K}$. As expected, this indicates that the biggest clusters and the slowest dynamics are found when the system is on the verge of magnetic order.

There are two reasons why the Griffiths phase is expected to be stabilized near the

Table 2. $\lambda$-values are obtained from our magnetization scaling in the limit $H \rightarrow 0$. The values are consistent with values of $\lambda$ obtained from specific heat and dc susceptibility measurements.

\begin{tabular}{llll}
\hline Sample & Specific heat $^{\mathrm{a}}$ & DC susceptibility $^{\mathrm{a}}$ & Magnetization scaling \\
\hline$x=0.7$ & - & - & $0.80 \pm 0.02$ \\
$x=1.0$ & 0.72 & 0.72 & $0.66 \pm 0.03$ \\
$x=1.5$ & 0.81 & 0.78 & $0.74 \pm 0.02$ \\
\hline
\end{tabular}

${ }^{a}$ Reference [20]. 
$x=x_{c}=1, T=0$ critical point in $\mathrm{UCu}_{5-x} \mathrm{Pd}_{x}$. In the antiferromagnetic phase, $x \leqslant 1$, the almost homogeneous, magnetically ordered background is increasingly broken up into finite-sized magnetic clusters as the Pd concentration is increased towards the critical value $x_{c}=1$. This would tend to stabilize the Griffiths phase with increasing $x<x_{c}$. However, as the Pd concentration is raised above $x_{c}$, the probability of finding Pd-deficient, magnetically ordered clusters decreases as the system is tuned away from the antiferromagnetic phase. Consequently, the Griffiths phase must ultimately be suppressed for $x>x_{c}$.

In addition to this general scenario, which would be applicable to any magnetically ordered system with a $T=0$ fixed point, there is another mechanism for the stabilization of the Griffiths phase at $x_{c}=1$ specific to $\mathrm{nFL}$ compounds having the $\mathrm{AuBe}_{5}$ crystal structure such as $\mathrm{UCu}_{5-x} \mathrm{Pd}_{x}$. The $\mathrm{AuBe}_{5}$ crystal structure has two inequivalent $\mathrm{Cu}$ sites, the $\mathrm{Cu}-\mathrm{I}$ and $\mathrm{Cu}$-II sites, whose occupancies per unit cell are, respectively, one and four. Structural and compositional studies $[13,14,21]$ reveal a preferential occupation of the $\mathrm{Cu}-\mathrm{I}$ site by $\mathrm{Pd}$ for $x \leqslant 1$. Consequently, $x=1$ would be expected to be an ordered line compound with complete filling of the $\mathrm{Cu}-\mathrm{I}$ site by $\mathrm{Pd}$, although a recent EXAFS measurement [14] finds $\sim 25 \% \mathrm{Cu}-\mathrm{Pd}$ disorder on the $\mathrm{Cu}-\mathrm{I}$ site. The implication is that the native $\mathrm{Cu}-\mathrm{Pd}$ site disorder and vacancies present in $\mathrm{UCu}_{4} \mathrm{Pd}$ are sufficient to stabilize the Griffiths phase. If the Pd concentration is either increased or decreased with respect to $x=1$, disorder increases, resulting in smaller clusters with faster reorientations, and thus to the suppression of the Griffiths phase. We believe that the coincidence of having the greatest degree of atomic order at the critical concentration $x_{c}=1$ ultimately stabilizes the Griffiths phase, which we propose dominates the low-temperature behaviour near the fixed point.

To summarize, our measurements have delineated a region of the composition-temperature phase diagram near the $T=0$ critical point in $\mathrm{UCu}_{5-x} \mathrm{Pd}_{x}$ in which even small amounts of disorder strongly affect the development of spatial and temporal magnetic correlations. We have shown that this proposed Griffiths phase is superimposed on a second nFL region, which is more robust under compositional variations and inhabits a more extensive part of the $(x, T)$ phase diagram. The implication of our work is that the true critical behaviour associated with the $T=0$ critical point is found in this second $n F L$ region. That is, the line $T^{*}(x)$ separates a higher-temperature region in which the length scales of the magnetic correlations are controlled purely by proximity to the $T=0$ critical point, from a lower-temperature region in which the correspondence between length scales and dynamics is influenced by disorder, albeit in a subtle way. The overall scale for $T^{*}(x)$ is determined not only by the degree of disorder present, but also by how effectively this disorder is coupled to the magnetism, and perhaps most importantly, by the intra- and inter-ion energy scales of the system. It is reasonable to expect substantial variation in $T^{*}(x)$ among the nFL systems already identified, suggesting that measurements on similar temperature ranges cannot be considered on an equal footing for different systems. We propose here an experimental solution to this problem, by outlining a general procedure for identifying the separatrix $T^{*}(x)$.

\section{Acknowledgments}

We are pleased to acknowledge helpful and stimulating conversations with A Castro Neto, B A Jones, G Kotliar, H von Löhneysen, D E MacLaughlin, L Sander, and S Sachdev. We would like to thank C Wolters, and, especially, M Shepard, for timely and generous technical assistance. Research at UCSD was supported by the National Science Foundation under Grant No DMR-97-0545. Work at the National High Magnetic Field Laboratory is supported by the National Science Foundation and the State of Florida through Cooperative Agreement No DMR-9527035. 


\section{References}

[1] Coleman P, Maple M B and Millis A J (ed) 1996 Institute for Theoretical Physics Conf. on Non-Fermi Liquid Behavior in Metals (Santa Barbara, CA, 1996); J. Phys.: Condens. Matter 8

[2] Maple M B, de Andrade M C, Herrmann J, Dalichaouch Y, Gajewski D A, Seaman C L, Chau R, Movshovich R, Aronson M C and Osborn R 1995 J. Low Temp. 99223

Maple M B, Dickey R P, Herrmann J, de Andrade M C, Freeman E J, Gajewski D A and Chau R 1996 J. Phys.: Condens. Matter 89743

[3] Bernal O O, MacLaughlin D E, Lukefahr H G and Andraka B 1995 Phys. Rev. Lett. 752023

MacLaughlin D E, Bernal O O and Lukefahr H G 1996 J. Phys.: Condens. Matter 89855

[4] Miranda E, Dobrosavljevic V and Kotliar G 1996 Physica 230569

[5] Thill M J and Huse D A 1995 Physica A 214321

[6] Castro Neto A H, Castilla G and Jones B A 1998 Phys. Rev. Lett. 813531

[7] Tsvelik A M and Reizer M 1993 Phys. Rev. B 489887

[8] Aronson M C, Maple M B, de Sa P, Tsvelik A M and Osborn R 1997 Europhys. Lett. 40245

[9] Andraka B and Stewart G R 1993 Phys. Rev. B 473208

[10] von Löhneysen H, Pietrus T, Portisch G, Schlager H G, Schroder A, Sieck M and Trappmann T 1994 Phys. Rev. Lett. 723262

[11] Chau R and Maple M B 1996 J. Phys.: Condens. Matter 89939

[12] Vollmer R, Pietrus T, von Löhneysen H, Chau R and Maple M B 1997 Physica B 230-232 600

[13] Chau R, Maple M B and Robinson R A 1998 Phys. Rev. B 58139

[14] Booth C H, MacLaughlin D E, Heffner R H, Chau R, Maple M B and Kwei G H 1998 Phys. Rev. Lett. 813960

[15] Aronson M C, Osborn R, Robinson R A, Lynn J W, Chau R, Seaman C L and Maple M B 1995 Phys. Rev. Lett. 75725

[16] Affleck I and Ludwig A W W 1993 Phys. Rev. B 487297

[17] Doniach S 1977 Valence Instabilities and Related Narrow Band Phenomena ed R D Parks (New York: Plenum) p 169

Doniach S 1977 Physica B 91231

[18] Lukefahr H G, Bernal O O, MacLaughlin D E, Seaman C L, Maple M B and Andraka B 1995 Phys. Rev. B 52 3038

[19] Griffiths R B 1969 Phys. Rev. Lett. 2317

[20] de Andrade M C, Chau R, Dickey R P, Dilley N R, Freeman E J, Gajewski D A, Maple M B, Movshovich R, Castro Neto A H, Castilla G E and Jones B A 1998 Phys. Rev. Lett. 81

[21] Troc R, Tran V H and Zolnierek Z J. Magn. Magn. Mater. 90+91 405

Zolnierek Z, Troc R and Tran V H 1990 Physica B 163727 